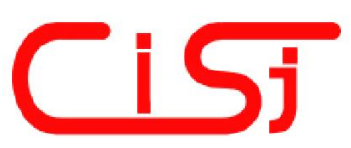

\title{
COMPUTING UNCERTAINTY OF THE EXTREME VALUES IN RANDOM SAMPLES
}

\author{
Mykhaylo Dorozhovets ${ }^{1), 2)}$, Ivanna Bubela ${ }^{2)}$ \\ 1) Rzeszow University of Technology, W. Pola str., 2A, 35-959, Rzeszow, Poland, email: michdor@prz.edu.pl \\ ${ }^{2)}$ National University - Lviv Polytechnic, Bandera str., 12, 79013, Lviv, Ukraine, email: popovych.i@ukr.net
}

\begin{abstract}
This paper proposes and analyses a statistical method for uncertainty evaluation of extreme values (minimal or maximal) for measurement results with significantly limited number of observations $n=3 \ldots 10$ and considerable deviation of observation probability density function (PDF) from normal distribution. The method is based on properties of order statistics. It can be used for the uncertainty evaluation of mechanical properties of testing products in a food industry (when minimal values of measurement results are observed) and for the investigation of a number of harmful elements (when maximal values of measurement results are observed). Copyright (C) Research Institute for Intelligent Computer Systems, 2016. All rights reserved.
\end{abstract}

Keywords: measurement, extreme values, minimal value of observations, maximal value of observations, uncertainty, distribution.

\section{INTRODUCTION}

Control of technological processes parameters in manufacturing products and control of measurement processes is an integral element of system designed to detect or prevent output of defective products on output and to protect the company from poor quality materials. The final aim of control is to obtain accurate results on the basis of conformity of the products and processes with the requirements of regulatory and technical documentation and standards is established. Evaluation of the uncertainty of measurement results is a necessary component during the control [1].

In some cases a minimal or maximal value of observations is the measurement result, and uncertainty of this value should be found. Recommendation as to its estimation is not given in GUM [1].

This paper gives a general theoretical approach to computing uncertainties of test measurements results, in which the minimal or maximal value in random sample of several observations is an informative parameter. Investigation results are given for the method when the probability density function (PDF) of the population does not contradict normal distribution, Laplace, uniform, arcsine, Cauchy or Flatten-Gaussian (it's convolution of normal and uniform [2, 3]).

The PDF of maximal value is symmetrical to the PDF of minimal value. That's why parameters of uncertainty of maximal value can be calculated in the same way as for minimal value. But the opposite sign of the maximal value deviation from the expected value should be taken into account.

\section{THEORY OF EXTREME VALUES UNCERTAINTIES}

Testing of the quality control of plastic tubes is considered to be an example of putting these theoretical backgrounds into practice. In this test two parameters are measured - percent elongation and tensile strength of the plastic tube in the process of its rupture $[4,5,6,7,8]$. According to the test requirements $[9,10,11]$, the minimum values of the percent elongation at break and tensile strength at yield are calculated rounded to the second significant digit.

Problem of computing uncertainty component of the minimal value by statistical method (type A) in percent elongation and tensile strength tests, as noted above, minimum values of the test specimens parameters have to be found. Therefore, it is impossible to apply directly the GUM method of measurements uncertainty evaluation with multiple observations [1].

As an example computing of the uncertainty of minimum values of controlled parameters from the sample of five elements is performed $[4,5,6,7,8]$. The minimal observation $x_{\min }=x_{(I)}=\min \left(x_{1}, x_{2}, \ldots\right.$, $\left.x_{n}\right)$ is the first one from the set of ordered observations: $x_{(1)} \leq x_{(2)} \leq x_{(3)} \leq \ldots \leq x_{(n)}$. The result of 
a test measurement is not as usual the arithmetic mean $(\bar{x})$ but the minimal (or maximal) value of observations. Then, the standard and expanded uncertain-ties of test results cannot be computed according to standard GUM procedures [1]. Another procedure should be used.

It is obvious that minimal value is a random value, however its probability density function (PDF) is not equal to PDF $p(x)$ of population.

In the next sections minimal observation $x_{(1)}$ is denoted by $x_{1}$. Theoretical distribution $p\left(x_{1}\right)$ of minimal value $x_{1}$ for the normally distributed observations $(m=0, \sigma=1)$

$$
\begin{aligned}
& p\left(x_{1}\right)=\frac{1}{\sqrt{2 \pi}} \exp \left(-x^{2} / 2\right), \\
& F\left(x_{1}\right)=\frac{1}{\sqrt{2 \pi}} \int_{-\infty}^{x_{1}} \exp \left(-x^{2} / 2\right) d x
\end{aligned}
$$

can be described [12] by formula:

$$
p\left(x_{1}\right)=n \cdot \frac{1}{\sqrt{2 \pi}} \exp \left(-x^{2} / 2\right) \cdot\left[1-F\left(x_{1}\right)\right]^{n-1} .
$$

This distribution for $n=5$ and $n=10$ is presented in Fig. 1.
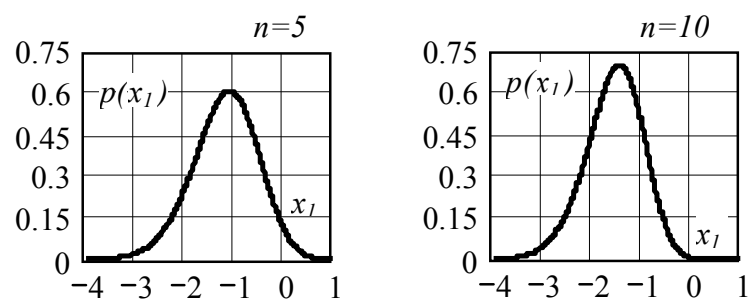

Fig. 1 - Distributions of minimal observation $x_{1}(n=5$ and $n=10$ )

From (1) the expected value $m_{01}$ of $x_{1}$ can be calculated as follows:

$$
m_{0,1}=\int_{-\infty}^{\infty} x_{1} p\left(x_{1}\right) d x_{1}
$$

and $\sigma_{01}$ standard deviation of the minimal observation:

$$
\sigma_{0,1}=\int_{-\infty}^{\infty} x_{1}^{2} p\left(x_{1}\right) d x_{1}-m_{0,1}^{2}
$$

The values $m_{01}$ (2) and $\sigma_{01}$ (3) for number of observation $n=3 \ldots 10$ and for normal distribution,

\begin{tabular}{|c|c|c|c|c|c|}
\hline$m_{01}$ & $\sigma_{01}$ & & $n$ & \multirow{2}{*}{$=\frac{\boldsymbol{m}_{\boldsymbol{0} \mathbf{1}}}{-0,79550}$} & \multirow{2}{*}{\begin{tabular}{|c|}
$\boldsymbol{\sigma}_{\boldsymbol{0 1}}$ \\
0,84111 \\
\end{tabular}} \\
\hline$-0,84628$ & 0,74798 & & 3 & & \\
\hline 1,02938 & 0,70122 & 象 & 4 & \multirow{2}{*}{$\begin{array}{l}-0,97964 \\
-1,12327\end{array}$} & 0,84904 \\
\hline 1,16296 & 0,66898 & \multirow{6}{*}{ 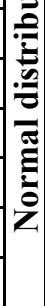 } & 5 & & 0,85739 \\
\hline 1,26721 & 0,64492 & & 6 & $-1,24186$ & 0,86428 \\
\hline 1,35218 & 0,62603 & & 7 & $-1,34313$ & 0,86972 \\
\hline 1,42360 & 0,61065 & & 8 & $-1,43162$ & 0,87403 \\
\hline$-1,48501$ & 0,59779 & & 9 & 1,51023 & 0,87748 \\
\hline 1,53875 & 0,58681 & & 10 & $-1,58095$ & 0,88030 \\
\hline$m_{01}$ & $\sigma_{01}$ & & $n$ & $m_{01}$ & $\sigma_{01}$ \\
\hline$-0,84628$ & 0,74798 & ฮิ & 3 & $-0,85217$ & 0,73289 \\
\hline 1,02938 & 0,70122 & s & 4 & $-1,03425$ & 0,67599 \\
\hline$-1,16296$ & 0,66898 & .. & 5 & : $-1,16534$ & 0,63606 \\
\hline 1,26721 & 0,64492 & 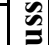 & 6 & $-1,26640$ & 0,60616 \\
\hline$-1,35218$ & 0,62603 & 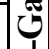 & 7 & -1,34791 & 0,58273 \\
\hline$-1,42360$ & 0,61065 & పี & 8 & $-1,41579$ & 0,56375 \\
\hline$-1,48501$ & 0,59779 & $\bar{\pi}$ & 9 & $-1,47369$ & 0,54798 \\
\hline 1,53875 & 0,58681 & & 10 & $-1,52400$ & 0,53461 \\
\hline
\end{tabular}
Laplace, uniform, arcsine, Cauchy or Flatten-

\begin{tabular}{|c|c|c|c|c|c|c|}
\hline$m_{01}$ & $\sigma_{01}$ & & $n$ & & $m_{01}$ & $\sigma_{01}$ \\
\hline$-0,86091$ & 0,70363 & \multirow[t]{2}{*}{$\mathrm{S}$} & 3 & & $-0,86601$ & 0,67136 \\
\hline$-1,03977$ & 0,62563 & & 4 & $\theta$ & $-1,03935$ & 0,56671 \\
\hline$-1,16457$ & 0,57005 & \multirow{3}{*}{$\begin{array}{c}\text {. } \\
0 \\
0 \\
0 \\
0\end{array}$} & 5 & $\doteq$ & $-1,15502$ & 0,48942 \\
\hline$-1,25804$ & 0,52875 & & 6 & 2 & $-1,23774$ & 0,43046 \\
\hline$-1,33155$ & 0,49696 & & 7 & 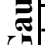 & $-1,29986$ & 0,38416 \\
\hline$-1,39145$ & 0,47177 & \multirow{3}{*}{ 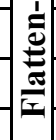 } & 8 & 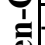 & $-1,34825$ & 0,34693 \\
\hline$-1,44159$ & 0,45132 & & 9 & \pm & $-1,38702$ & 0,31637 \\
\hline$-1,48445$ & 0,43438 & & 10 & & $-1,41880$ & 0,29086 \\
\hline
\end{tabular}
Gaussian, are presented in Table 1.

\begin{tabular}{|c|c|c|c|c|c|}
\hline$m_{01}$ & $\sigma_{01}$ & & $n$ & $m_{01}$ & $\sigma_{01}$ \\
\hline 0,86603 & 0,67082 & $\Xi$ & 3 & $=-0,85974$ & 0,64252 \\
\hline 1,03923 & 0,56569 & 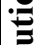 & 4 & $-1,02260$ & 0,50819 \\
\hline$-1,15470$ & 0,48795 & 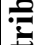 & 5 & $-1,12360$ & 0,40882 \\
\hline$-1,23718$ & 0,42857 & 3 & 6 & 㦹-1,19036 & 0,33460 \\
\hline$-1,29904$ & 0,38188 & 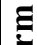 & 7 & :-1,23670 & 0,27820 \\
\hline 1,34715 & 0,34427 & 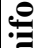 & 8 & -1,27012 & 0,23455 \\
\hline$-1,38564$ & 0,31334 & $\mathrm{D}$ & 9 & 《1,29499 & 0,20019 \\
\hline 1,41713 & 0,28748 & & 10 & $-1,31398$ & 0,17271 \\
\hline
\end{tabular}

Table 1. Expected numeric values $m_{01}$ and $\sigma_{01}$ of

\begin{tabular}{|c|c|c|c|}
\hline$m_{01}$ & $\sigma_{01}$ & & $n$ \\
\hline$-1,60218$ & 2,11374 & $=$ & 3 \\
\hline$-1,94208$ & 2,20644 & כ约 & 4 \\
\hline$-2,18491$ & 2,29652 & 象 & 5 \\
\hline$-2,36596$ & 2,37918 & 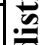 & 6 \\
\hline$-2,50288$ & 2,45533 & 2 & 7 \\
\hline$-2,60631$ & 2,52612 & 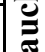 & 8 \\
\hline$-2,68339$ & 2,59226 & ש & 9 \\
\hline$-2,73926$ & 2,65420 & & 10 \\
\hline
\end{tabular}
minimal observation of $x_{1}$. 
If $m \neq 0$ and $\sigma \neq 1$ then expected value $m_{1}$ and standard deviation $\sigma_{l}$ of the minimal observation of $x_{1}$ are

$$
m_{1}=m+m_{0.1} \cdot \sigma ; \quad \sigma_{1}=\sigma_{0.1} \cdot \sigma .
$$

In practice the expected value $m_{1}$ of minimal observation $x_{l}$ is unknown, but after (4) the estimate $f_{1}$ for $m_{l}$ can be calculated as

$$
\hat{x}_{1}=\bar{x}+m_{0,1} \cdot s_{x},
$$

where arithmetical mean $\bar{x}$ and experimental standard deviation $s_{x}$ of observations are

$$
\begin{gathered}
\bar{x}=\frac{1}{n} \sum_{i=1}^{n} x_{i}, \\
s_{x}=\sqrt{\frac{1}{n-1} \sum_{i=1}^{n}\left(x_{i}-\bar{x}\right)^{2}} .
\end{gathered}
$$

Experimental standard uncertainty of minimal value calculated from (6) and (7) is

$$
u_{A}\left(x_{1}\right)=\sigma_{0.1} \cdot s_{x} \text {. }
$$

Distribution $p_{z l}\left(z_{l}\right)$ of the minimal observation $x_{I}$ deviation from mean $\bar{x}$, normalized to $s_{x}$ is

$$
z_{1}=\frac{x_{1}-\bar{x}}{s_{x}}
$$

This distribution does not depend on $\bar{x}$ and on $s_{x}$. It depends only on population distribution $p(x)$ and number of observations $n$. It can be shown that the range of random value $z_{1}$ is independent of population $\mathrm{PDF}$ and equals to

$$
-(n-1) / \sqrt{n} \leq z_{1} \leq-1 / \sqrt{n} .
$$

Distribution $p_{z l}\left(z_{1}\right)$ consists of $n-1$ sections, with bounds $z_{b, i}(i=1,2, \ldots n-1)$ that are determined by the formula:

$$
\begin{aligned}
& z_{b, i}=-\sqrt{(n-1)(n-i) /(n \cdot i)}, \\
& i=1,2, \ldots, n-1
\end{aligned}
$$

In test procedure the minimal observation $x_{1}$ is compared with the critical value $x_{\text {critic }}$, then after determination of $x_{1}$, the left-hand side of expanded uncertainty $U_{p, \text { low }}\left(x_{1}\right)$ should be calculated as follows:

$$
x_{1}-U_{p, \text { low }}\left(x_{1}\right) \geq x_{\text {critic }} .
$$

For the very small number of observations (for example $n=5$ ) the most important is the first part (left side) with bounds

$$
\begin{aligned}
& z_{b, 1}=-(n-1) / \sqrt{n}, \\
& z_{b, 2}=-\sqrt{(n-1)(n-2) / 2 n} .
\end{aligned}
$$

If $n=5$ from (13) then

$$
\begin{aligned}
& z_{b, 1}=-4 / \sqrt{5} \approx-1,7889 ; \\
& z_{b, 2}=-\sqrt{6 / 5} \approx-1,0954,
\end{aligned}
$$

because at the end of the first part the cumulative function is

$$
F_{z 1}\left(z_{1}\right)=\int_{-(n-1) / \sqrt{n}}^{-\sqrt{(n-1)(n-2) / 2 n}} p_{z 1}\left(z_{1}\right) d z_{1}>0,10 .
$$

For normally distributed $n=5$ observations, the theoretical distribution $p_{z l}\left(z_{l}\right)$ at the left-hand side can be described as

$$
\begin{aligned}
& p_{z 1}\left(z_{1}\right)=\frac{5 \sqrt{5}}{2 \pi} \sqrt{1-\frac{5}{16} z_{1}^{2}}, \\
& -\frac{4}{\sqrt{5}} \leq z_{1} \leq-\sqrt{\frac{6}{5}} .
\end{aligned}
$$

From (15) cumulative function in this part is

$$
\begin{gathered}
F_{z 1}\left(z_{1}\right)=\int_{-\sqrt{2}}^{z_{1}} p_{z 1}\left(z_{1}\right) d z_{1}= \\
=\frac{5}{2}\left[\frac{1}{2 \pi} \cdot z_{1} \sqrt{5-\left(\frac{5}{4} z_{1}\right)^{2}}+\frac{2}{\pi} \arcsin \left(\frac{\sqrt{5}}{4} z_{1}\right)+1\right] .
\end{gathered}
$$

For $z_{1}=-\sqrt{6 / 5}$ the cumulative function is $F_{Z 1}(-\sqrt{6 / 5})=0,6806$. Total distribution $p_{z l}\left(z_{1}\right)$ of $z_{1}$ is shown in Fig. 2 .

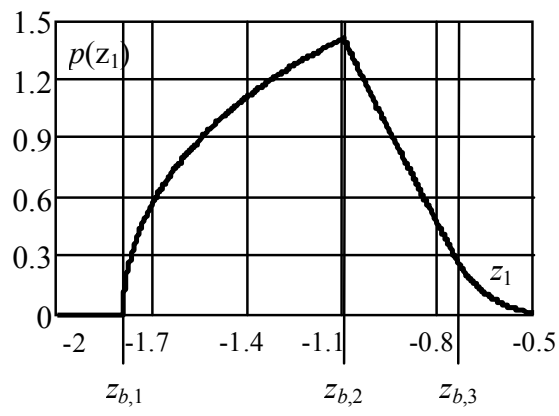

Fig. 2 - Distribution of normalized deviation $\mathrm{z}_{1}(n=5)$ 
The lower $k_{\text {low }}(p)$ coverage factor for the confidence level $p$ can be calculated from equation:

$$
\int_{-2}^{k_{\text {low }}(n, p)} p_{z 1}\left(z_{1}\right) d z_{1}=F_{z 1}\left(z_{1}\right)=1-p
$$

The values $k_{\text {low }}(5, p)$ for $p=0,90 ; 0,95 ; 0,975$; 0,99 and 0,995 and for $n=5$ are presented in Table 2 .

Table 2. Numeric values of coverage factors.

\begin{tabular}{|c|c|c|c|c|c|}
\hline $\boldsymbol{p}$ & $\mathbf{0 , 9 0}$ & $\mathbf{0 , 9 5}$ & $\mathbf{0 , 9 7 5}$ & $\mathbf{0 , 9 9}$ & $\mathbf{0 , 9 9 5}$ \\
\hline $\boldsymbol{k}_{\text {low }}(\mathbf{5 , \boldsymbol { p } )}$ & $-1,6016$ & $-1,6714$ & $-1,7156$ & $-1,7489$ & $-1,7637$ \\
\hline
\end{tabular}

From (9) and Table 2 the lower limit $U_{I-p, l o w}\left(x_{1}\right)$ of expanded uncertainties of minimal value is

$$
x_{1, p}=\bar{x}+k_{\text {low }}(n, p) \cdot s_{x} .
$$

\section{SIMULATIONS BY MONTE CARLO METHOD}

Analytical research of efficiency of the method proposed for evaluating measurement result and its standard uncertainty was investigated by Monte Carlo (MC) method. During the research the following basic normalized distributions $(m=0, \sigma=1)$ of the population have been accepted: normal, Laplace, uniform, arcsine and Cauchy; number of observations $n=3,4,5,6,7,8,9,10$; number of realizations is $\mathrm{M}=10^{5}$.

Perform generate the $j=1,2, \ldots, M=10^{5}$ to $n=3,4,5,6,7,8,9,10$ independent random results with different distributions.

For the every observation $n=3,4,5,6,7,8,9$, 10 the minimal result is determined by the formula:

$$
x 1_{n, j}=\min \left(x_{n, j}\right)
$$

The arithmetical mean value $\overline{x_{n, j}}$ from (6) and experimental standard deviation $s_{n, j}$ from (7) for each group of $n$ observations is calculated.

Based on the obtained values $\overline{x_{n, j}}$ and $s_{n, j}$ deviation $z 1_{j}$ of the minimal result $x 1_{n, j}$ from the mean is calculated from (9).

Statistical processing of the obtained results is performed:

- deviation $z 1_{j}$ mean value of the minimal result from the mean is calculated as

$$
\overline{z 1}=\frac{1}{M} \sum_{j-1}^{M} z 1_{j}
$$

- estimate of the minimal result standard deviation is calculated as

$$
S_{z 1}=\sqrt{\frac{1}{M-1} \sum_{j=1}^{M}\left(z 1_{j}-\overline{z 1}\right)^{2}} ;
$$

- maximal $\max (z 1)$ and minimal $\min (z 1)$ experimental values of deviation $z l_{j}$ of the minimal result from the mean.

All research results obtained according to the calculation formulas (20), (21) and others for

\begin{tabular}{|c|c|c|c|c|}
\hline$n$ & $\overline{z 1}$ & $s_{z 1}$ & $\max (z 1)$ & $\min (z 1)$ \\
\hline \multicolumn{5}{|c|}{ Normal distribution } \\
\hline 3 & 0,9543 & 0,1751 & 1,1547 & 0,5774 \\
\hline 4 & 1,1183 & 0,2302 & 1,5000 & 0,5030 \\
\hline 5 & 1,2376 & 0,2636 & 1,7888 & 0,4638 \\
\hline 6 & 1,3305 & 0,2846 & 2,0384 & 0,4494 \\
\hline 7 & 1,4083 & 0,3016 & 2,2599 & 0,4773 \\
\hline 8 & 1,4756 & 0,3135 & 2,4529 & 0,4891 \\
\hline 9 & 1,5313 & 0,3227 & 2,6294 & 0,5222 \\
\hline 10 & 1,5838 & 0,3319 & 2,7563 & 0,5923 \\
\hline \multicolumn{5}{|c|}{ Uniform distribution } \\
\hline 3 & 0,9509 & 0,1813 & 1,1547 & 0,5774 \\
\hline 4 & 1,1045 & 0,2345 & 1,5000 & 0,5042 \\
\hline 5 & 1,2080 & 0,2619 & 1,7888 & 0,4653 \\
\hline 6 & 1,2824 & 0,2753 & 2,0379 & 0,4568 \\
\hline 7 & 1,3395 & 0,2827 & 2,2554 & 0,4783 \\
\hline 8 & 1,3854 & 0,2844 & 2,4470 & 0,4857 \\
\hline 9 & 1,4202 & 0,2837 & 2,6077 & 0,6038 \\
\hline 10 & 1,4504 & 0,2820 & 2,6910 & 0,6175 \\
\hline \multicolumn{5}{|c|}{ Laplace distribution } \\
\hline 3 & 0,9530 & 0,1776 & 1,1547 & 0,5774 \\
\hline 4 & 1,1207 & 0,2454 & 1,5000 & 0,5018 \\
\hline 5 & 1,2488 & 0,2942 & 1,7888 & 0,4585 \\
\hline 6 & 1,3530 & 0,3306 & 2,0391 & 0,4297 \\
\hline 7 & 1,4439 & 0,3620 & 2,2649 & 0,4620 \\
\hline 8 & 1,5259 & 0,3872 & 2,4688 & 0,4469 \\
\hline 9 & 1,5960 & 0,4079 & 2,6500 & 0,4417 \\
\hline 10 & 1,6639 & 0,4290 & 2,8333 & 0,4999 \\
\hline \multicolumn{5}{|c|}{ Arcsine distribution } \\
\hline 3 & 0,9433 & 0,1949 & 1,1547 & 0,5774 \\
\hline 4 & 1,0811 & 0,2585 & 1,5000 & 0,5004 \\
\hline 5 & 1,1714 & 0,2912 & 1,7888 & 0,4490 \\
\hline 6 & 1,2302 & 0,3067 & 2,0407 & 0,4103 \\
\hline 7 & 1,2742 & 0,3159 & 2,2663 & 0,4126 \\
\hline 8 & 1,3041 & 0,3165 & 2,4683 & 0,4436 \\
\hline 9 & 1,3244 & 0,3127 & 2,6444 & 0,4339 \\
\hline 10 & 1,3415 & 0,3077 & 2,7814 & 0,4950 \\
\hline \multicolumn{5}{|c|}{ Cauchy distribution } \\
\hline 3 & 0,9431 & 0,1951 & 1,1547 & 0,5774 \\
\hline 4 & 1,1066 & 0,3031 & 1,5000 & 0,5000 \\
\hline
\end{tabular}
normal, Laplace, uniform, arcsine and Cauchy distributions are given in Table 3.

Table 3. Results of investigation of the minimal value deviation $z 1_{j}$ from the mean. 


\begin{tabular}{|c|c|c|c|c|}
\hline $\mathbf{5}$ & 1,2379 & 0,3959 & 1,7889 & 0,4472 \\
\hline $\mathbf{6}$ & 1,3489 & 0,4777 & 2,0412 & 0,4083 \\
\hline $\mathbf{7}$ & 1,4502 & 0,5543 & 2,2678 & 0,3780 \\
\hline $\mathbf{8}$ & 1,5472 & 0,6223 & 2,4749 & 0,3536 \\
\hline $\mathbf{9}$ & 1,6329 & 0,6843 & 2,6667 & 0,3334 \\
\hline $\mathbf{1 0}$ & 1,7217 & 0,7469 & 2,8460 & 0,3163 \\
\hline
\end{tabular}

It was also investigated how often the proposed algorithm for the criterion of the residual sums of squares of test sample residual deviations from the model experiment correctly chooses the model distribution. One of the quantitative indicators of distribution densities mutual "proximity" is their contra-kurtosis $\zeta$ which is calculated as follows [13]:

$$
\zeta=1 / \sqrt{\varepsilon}
$$

where $\varepsilon$ is skewness of distribution kurtosis and is calculated as

$$
\varepsilon=\mu_{4} / \sigma^{4}
$$

Depending on the value of contra-kurtosis some typical distributions can be located as follows: 1Laplace $\zeta \mathrm{L}=0,408$, 2-normal $\zeta \mathrm{N}=0,577$, 3-uniform $\zeta \mathrm{R}=0,745$ and 4 -arcsine $\zeta$ Asin $=0,816,5$-Cauchy $\zeta \mathrm{K}=0$.

Table 4. The numeric values of MC experimental

\begin{tabular}{|c|c|c|c|c|c|c|}
\hline $\begin{array}{l}\text { contra- } \\
\text { kurtosis }\end{array}$ & skewness & \multirow{9}{*}{ 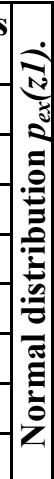 } & $n$ & \multirow{2}{*}{ 葡 } & \multirow{2}{*}{\begin{tabular}{|c|c|}
$\begin{array}{c}\text { contra- } \\
\text { kurtosis }\end{array}$ \\
0,7205
\end{tabular}} & \multirow{2}{*}{\begin{tabular}{|c|} 
skewness \\
$-0,5366$ \\
\end{tabular}} \\
\hline 0,702 & $-0,571$ & & 3 & & & \\
\hline 0,686 & $-0,216$ & & 4 & \multirow{7}{*}{ 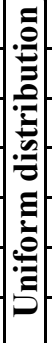 } & 0,6914 & $-0,1188$ \\
\hline 0,664 & $-0,021$ & & 5 & & 0,6524 & 0,1311 \\
\hline 0,645 & 0,115 & & 6 & & 0,6179 & 0,3028 \\
\hline 0,630 & 0,210 & & 7 & & 0,5899 & 0,4114 \\
\hline 0,616 & 0,267 & & 8 & & 0,5672 & 0,4964 \\
\hline 0,604 & 0,336 & & 9 & & 0,5484 & 0,5614 \\
\hline 0,597 & 0,358 & & 10 & & 0,5414 & 0,5790 \\
\hline $\begin{array}{l}\text { contra- } \\
\text { kurtosis }\end{array}$ & skewness & \multirow{6}{*}{ 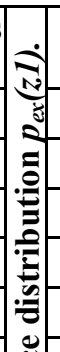 } & $n$ & \multirow{6}{*}{ 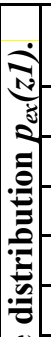 } & $\begin{array}{l}\text { contra- } \\
\text { kurtosis }\end{array}$ & skewness \\
\hline 0,7100 & $-0,5559$ & & 3 & & 0,7518 & $-0,4889$ \\
\hline 0,6999 & $-0,2567$ & & 4 & & 0,7069 & $-0,0458$ \\
\hline 0,6857 & $-0,1052$ & & 5 & & 0,6556 & 0,2176 \\
\hline 0,6742 & 0,0072 & & 6 & & 0,6079 & 0,4023 \\
\hline 0,6645 & 0,0898 & & 7 & & 0,5736 & 0,5362 \\
\hline 0,6540 & 0,1323 & : & 8 & \multirow{3}{*}{ 量 } & 0,5481 & 0,6155 \\
\hline 0,6443 & 0,1948 & 암 & 9 & & 0,5257 & 0,6858 \\
\hline 0,6396 & 0,2179 & - & 10 & & 0,5154 & 0,7162 \\
\hline
\end{tabular}
contra-kurtosis and skewness for distributions $p_{e x}(z 1)$.

\begin{tabular}{|c|c|c|c|}
\hline $\begin{array}{c}\text { contra- } \\
\text { kurtosis }\end{array}$ & skewness & \multirow{n}{*}{} & $\boldsymbol{n}$ \\
\hline 0,7527 & $-0,4879$ & $\mathbf{3}$ \\
\hline 0,7368 & $-0,3127$ & & $\mathbf{4}$ \\
\hline 0,7327 & $-0,2308$ & $\mathbf{5}$ & $\mathbf{5}$ \\
\hline 0,7329 & $-0,1734$ & $\mathbf{6}$ \\
\hline 0,7373 & $-0,1299$ & $\mathbf{7}$ \\
\hline 0,7368 & $-0,1189$ & $\mathbf{8}$ \\
\hline 0,7376 & $-0,0931$ & $\mathbf{9}$ \\
\hline 0,7417 & $-0,0891$ & $\mathbf{1 0}$ \\
\hline
\end{tabular}

The deviations $\mathrm{z} 1_{j}$ of Fig. 3 shows the histograms of the minimal result from the mean value at $n=3$, $4,5,6,7,8,9,10$ for normal, Laplace, uniform, arcsine and Cauchy distributions.

Table 5 gives as an example the values of upper $z l_{\text {up }}$ and lower $z 1_{\text {low }}$ confidence limits for the deviation $z l_{j}$ of the minimal result from the mean value on the level of trust $p=1-\alpha$ under probability of $p=0,90(\alpha=0,1(10 \%) ; p=0,925(\alpha=0,075 \quad(7,5 \%))$; $p=0,95(\alpha=0,05(0,5 \%)) ; p=0,975(\alpha=0,025(2,5 \%))$ for $n=3,4,5,6,7,8,9,10$ for the normally distributed observations.

Table 5. Results of research of the upper $z 1_{u p}$ and lower $z 1_{\text {low }}$ confidence limits for the deviation $\mathrm{z} 1_{j}$ of the

\begin{tabular}{|c|c|c|c|c|}
\hline \multicolumn{5}{|c|}{ Normal distribution } \\
\hline$p$ & 0,90 & 0,925 & 0,95 & 0,975 \\
\hline$z 1_{u p}(3, p)$ & 1,1532 & 1,1538 & 1,1543 & 1,1546 \\
\hline$z 1_{\text {up }}(4, p)$ & 1,4626 & 1,4718 & 1,4809 & 1,4907 \\
\hline$z 1_{\text {up }}(5, p)$ & 1,6718 & 1,6932 & 1,7166 & 1,7437 \\
\hline$z 1_{u p}(6, p)$ & 1,8211 & 1,8511 & 1,8848 & 1,9319 \\
\hline$z 1_{\text {up }}(7, p)$ & 1,9386 & 1,9753 & 2,0196 & 2,0814 \\
\hline$z 1_{u p}(8, p)$ & 2,0333 & 2,0757 & 2,1290 & 2,2015 \\
\hline$z 1_{\text {up }}(9, p)$ & 2,1120 & 2,1582 & 2,2170 & 2,3019 \\
\hline$z 1_{u p}(10, p)$ & 2,1780 & 2,2298 & 2,2932 & 2,3866 \\
\hline$z 1_{\text {low }}(3, p)$ & 0,6284 & 0,6157 & 0,6024 & 0,5902 \\
\hline$z 1_{\text {low }}(4, p)$ & 0,7288 & 0,7023 & 0,6680 & 0,6218 \\
\hline$z 1_{l o w}(5, p)$ & 0,8094 & 0,7822 & 0,7462 & 0,6926 \\
\hline$z 1_{\text {low }}(6, p)$ & 0,8827 & 0,8526 & 0,8146 & 0,7561 \\
\hline$z 1_{\text {low }}(7, p)$ & 0,9409 & 0,9104 & 0,8707 & 0,8129 \\
\hline$z 1_{l o w}(8, p)$ & 0,9955 & 0,9632 & 0,9240 & 0,8628 \\
\hline$z 1_{l o w}(9, p)$ & 1,0438 & 1,0125 & 0,9735 & 0,9111 \\
\hline$z 1_{l o w}(10, p)$ & 1,0840 & 1,0524 & 1,0114 & 0,9467 \\
\hline
\end{tabular}
minimal result for the normal distribution.

Fig. 4 shows the upper $z 1_{u p}$ and lower $z 1_{\text {low }}$ confidence limits for the deviation $z l_{j}$ of the minimal result from the mean value under probability of $p=0,90, p=0,925, p=0,95, p=0,975$ of $n=3,4,5,6$, $7,8,9,10$ for normal-1, uniform-2, Laplace-3, arcsine- 4 and Cauchy- 5 distributions. 

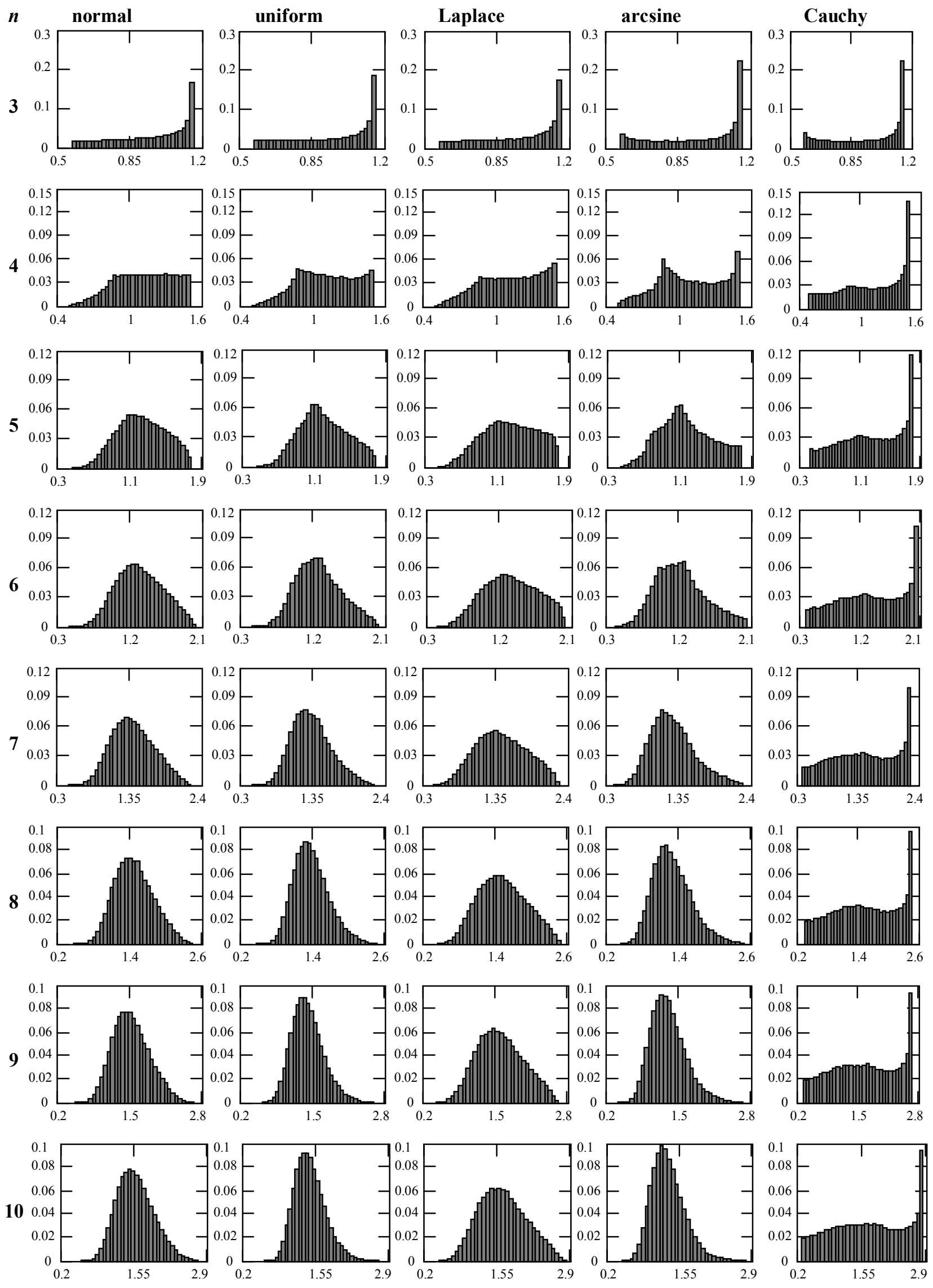

Fig. 3 - Histograms of the deviation $z 1_{j}$ 

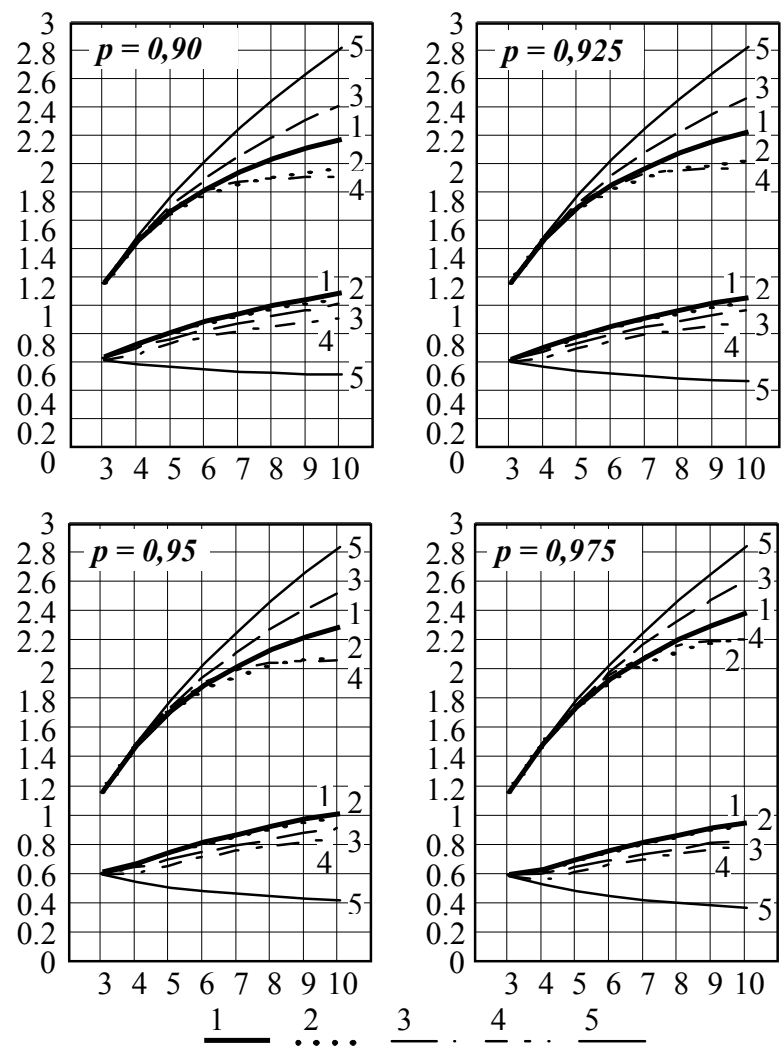

Fig. 4 - The upper $z 1_{u p}$ and lower $z 1_{l o w}$ confidence limits for the deviation $z 1_{j}$ of the minimal result

Table 6 shows as an example one-sided $z 1_{o}$ confidence limits for the deviation $z 1_{j}$ of the minimal result from the mean value if $p=0,90, p=0,925$, $p=0,95, p=0,975$ for $n=3,4,5,6,7,8,9,10$ and for the normal distribution.

Table 6. Results of research of the one-sided $z 1_{o}$ confidence limits for the deviation $\mathrm{z}_{j}$ of the minimal result for the normal distribution.

\begin{tabular}{|c|c|c|c|c|}
\hline \multicolumn{5}{|c|}{ Normal distribution } \\
\hline$p$ & 0,90 & 0,925 & 0,95 & 0,975 \\
\hline$z 1_{o}(3, p)$ & 1,1485 & 1,1513 & 1,1532 & 1,1543 \\
\hline$z 1_{o}(4, p)$ & 1,4253 & 1,4439 & 1,4626 & 1,4809 \\
\hline$z 1_{o}(5, p)$ & 1,6021 & 1,6338 & 1,6718 & 1,7166 \\
\hline$z 1_{o}(6, p)$ & 1,7271 & 1,7690 & 1,8211 & 1,8848 \\
\hline$z 1_{o}(7, p)$ & 1,8281 & 1,8780 & 1,9386 & 2,0196 \\
\hline$z 1_{o}(8, p)$ & 1,9078 & 1,9628 & 2,0333 & 2,1290 \\
\hline$z 1_{o}(9, p)$ & 1,9772 & 2,0372 & 2,1120 & 2,2170 \\
\hline$z 1_{o}(10, p)$ & 2,0388 & 2,1012 & 2,1780 & 2,2932 \\
\hline
\end{tabular}

Fig. 5 shows the one-sided $z 1_{o}$ confidence limits for the deviation $z 1_{j}$ of the minimal result from the mean value if $p=0,90, p=0,925, p=0,95, p=0,975$ for $n=3,4,5,6,7,8,9,10$ and for normal-1, uniform-2, Laplace-3, arcsine-4 and Cauchy-5 distributions.
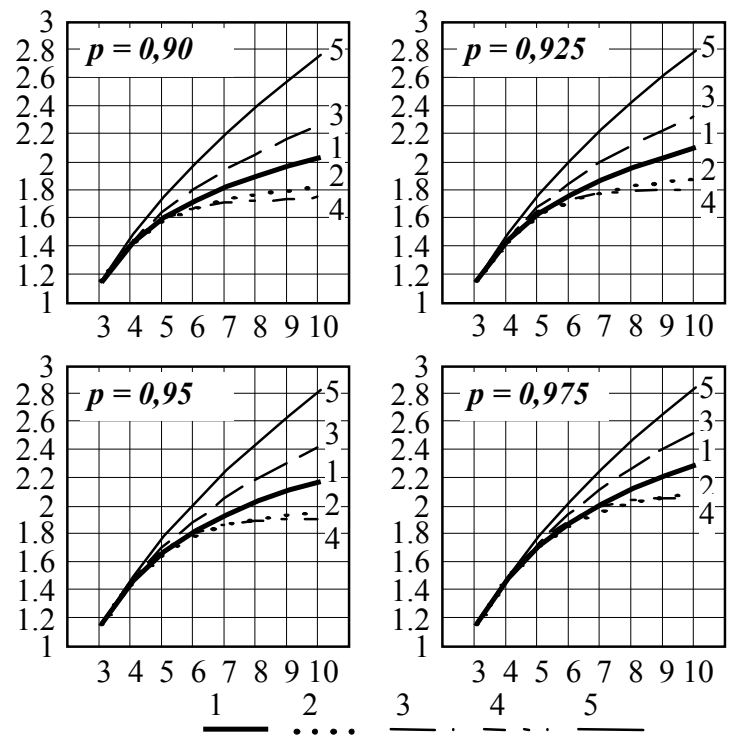

Fig. 5 - The one-sided $z 1_{o}$ confidence limits for the deviation $\mathrm{z}_{j}$ of the minimal result for the

distributions: 1-normal; 2-uniform; 3-Laplace; 4arcsine; 5-Cauchy

Fig. 6 shows in percentage form difference between deviations of the upper $z 1_{u p}$ and lower $z 1_{\text {low }}$ confidence limits for the deviation $z 1_{j}$ of the minimal result from the mean value and the normal distribution under probability of $p=0,90, p=0,925$, $p=0,95, p=0,975$ of $n=3,4,5,6,7,8,9,10$ for uniform-2, Laplace-3, arcsine-4 distributions.
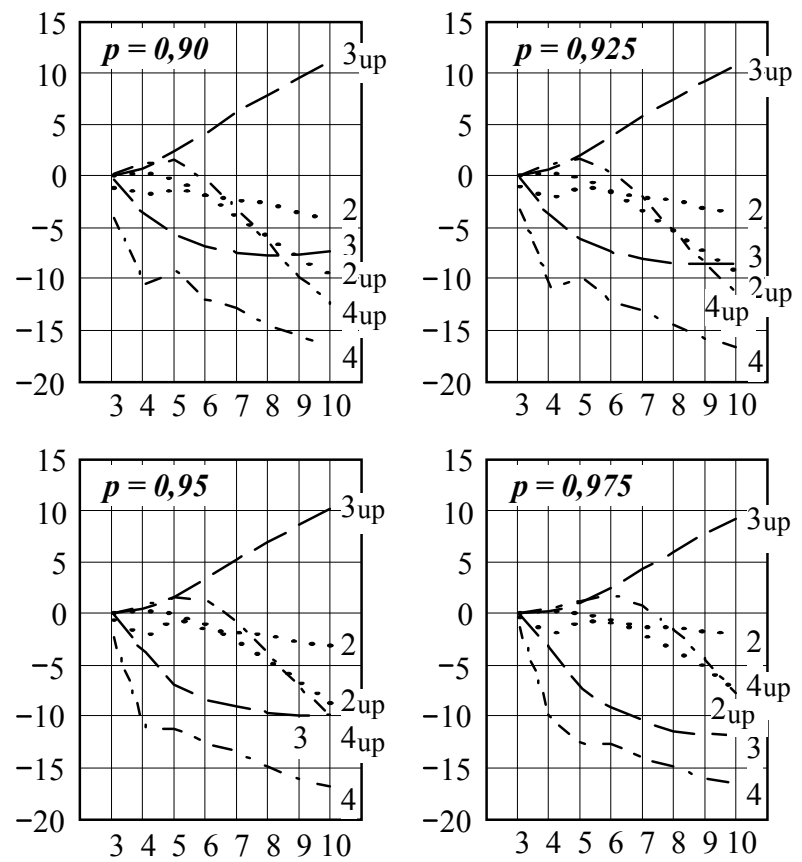

$$
\text { 2... } 3 \text {. } 4 \text {. }
$$

Fig. 6 - The difference between deviations of the upper $z 1_{u p}$ and lower $z 1_{\text {low }}$ confidence limits for the deviation $\mathrm{z} 1_{j}$ of the minimal result from the mean value and the normal distribution (uniform-2, Laplace-3, arcsine-4) 


\section{CONCLUSION}

As the PDF of maximal value is symmetrical to the PDF of minimal value, parameters of uncertainty of maximal value can be calculated in the same way as uncertainty of minimal value [14]. Only the opposite sign of the deviation of maximal value from the expected value should be taken into account.

Theoretically, for an arbitrary distribution of observations $p_{e x}(z 1)$ the deviation $z l$ which is relative to the standard deviation of minimum observation from the mean value is in the range of $-(n-1) / \sqrt{n} \leq z_{1} \leq-1 / \sqrt{n}$.

From Fig. 6 we can see, that for all studied PDF and if number of observations is limited, for example $n \leq 4,5$, the value of expansion coefficient deviates from the normal coefficient only about $\pm 10 \%$ and for $n=10$ is very close to $\pm 15 \%$.

Therefore, in case a priori PDF of observations is unknown and number of them is small $(n \leq 4,5)$ then a normal distribution value of expansion coefficient, can be used to calculate expanded uncertainty. For example, if $n=5$ then for all distributions expansion coefficient can be calculated using the formulas (16) and (17).

\section{REFERENCES}

[1] Guide to the Expression of Uncertainty in Measurement, First ed. 1993 ISO Switzerland, last corrected ed. JCGM BIPM 100, 2008 and Supplement 1- Propagation of distributions using a Monte-Carlo method.

[2] M. Dorozhovets, I. Popovych, "Processing of the random observations with Flatten-Gaussian distribution by approximate order statistics method," in Proceedings of the IEEE 8th International Conference on Intelligent Data Acquisition and Advanced Computing Systems: Technology and Applications (IDAACS'2015), Warsaw, Poland, 24-26 September 2015, vol. 1, pp. 149-152.

[3] M. Dorozhovets, I. Popovych, "Processing of the random observations with Flatten-Gaussian distribution by approximate order statistics method," in Proceedings of the Ukrainian scientific-technical conference of young scientists in the field of metrology "Technical Using of Measurement 2015," Slavsko, Ukraine, February 1-5, 2016, pp. 119-121. (in Ukrainian).

[4] O. V. Avramenko, M. M. Dorozhovets, I. V. Popovych, "Evaluation of uncertainty of measurement results in testing of percent elongation and tensile strength of plastic products," Automation, Measurement and
Control, Lviv Polytechnic National University, 2014. (in Ukrainian).

[5] M. Dorozhovets, I. Popovych, Z. L. Warsza, "Method of evaluation the measurement uncertainty of the minimal value of observations and its application in testing of plastic products," Advanced Mechatronics Solutions, vol. 393 of the series Advances in Intelligent Systems and Computing, Springer International Publishing Switzerland, pp. 421430, 2015.

[6] M. Dorozhovets, Z. L. Warsza, I. Popovych, "Uncertainty evaluation of the minimal value measurements," Measurement Automation Monitoring, vol. 61, no. 08, pp. 395-398, August 2015.

[7] O. V. Avramenko, M. M. Dorozhovets, I. V. Popovych, "Evaluation of uncertainty of measurement results in testing of percent elongation and tensile strength of plastic products," in Proceedings of the Ukrainian scientific-technical conference of young scientists in the field of metrology "Technical Using of Measurement 2015," Slavsko, Ukraine, February 2-6, 2015, pp. 94-96. (in Ukrainian).

[8] M. Dorozhovets, I. Popovych, Z. Warsza, "Evaluation of the measurement uncertainty of the minimal value of observations," in Proceedings of the XI Scientific-Technical Conference on Problems and Progress in Metrology, Kościelisko, Poland, June 07-10, 2015, pp. 60-66.

[9] Tensile Testing, ASM International, Second Edition, 2004.

[10] D 638 Test Method for Tensile Properties of Plastics, Annual Book of ASTM Standards, Vol 08.01.

[11] GOST 11262-80, GOST 26277-84, GOST 12423-66, Ukraine standards of testing methods and conditions of plastic materials and products.

[12] M. Fisz, Probability Theory and Mathematical Statistics, John Willey \& Sons, London, 1963.

[13] P. V. Novitski, I. A. Zograf, Evaluation of measurement result errors, Leningrad, Energoatomizdat, 1985, 248 p. (in Russian).

[14] O. A. Botsiura, Yu. G. Zharko, I. P. Zakharov, "Measurement uncertainty evaluation of the maximum observed value of the test parameter," Information Processing Systems, issue 2(127), Kharkiv, pp. 21-23, 2015. (in Russian). 


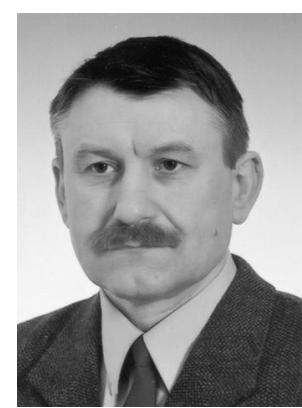

Mykhaylo Dorozhovets, Prof. dr hab. Eng. Graduate (1975) at the Department of Information and Measurement Technology, candidate of technical sciences $(P h D)$ in 1986 and defended Doctor of technical science (habilitation) in 2001. He is now professor in the Department of Metrology and Measurement Systems Rzeszów University of Technology and the National Ukra inian University "Lviv Politechnic".

$\mathrm{He}$ conducts research in the field of tomography, the measurement and signal processing, analysis and evaluation of the uncertainty of measurement results.

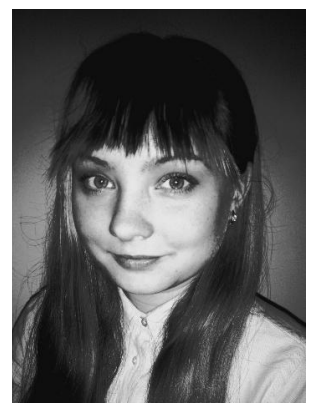

Ivanna Bubela, MSc. El. Eng. Graduated from National University of Ukraine "Lviv Polytechnic". Master degree in "Metrology and Measurement Technologies", Institute of Computer Technologies, Automation and Metrology in 2012. Currently: postgraduate Ph.D. student at National University Lviv Polytechnic. Ph.D. project deals with the processing of measurement results, which distribution differs from normal. 